\title{
MANEJO Y CONOCIMIENTOS SOBRE HEMODERIVADOS DE UN GRUPO DE PROFESIONALES DE ENFERMERÍA HOSPITALARIA DE MADRID
}

\author{
MANAGEMENT AND KNOWLEDGE ABOUT BLOOD PRODUCTS OF A GROUP OF PROFESSIONAL NURSING \\ AT A HOSPITAL IN MADRID \\ GESTÃO DO CONHECIMENTO E PRODUTOS DERIVADOS DO SANGUE UM GRUPO DE ENFERMAGEM DO \\ HOSPITAL PROFISSIONAL DE MADRID
}

\author{
Rafael Muñoz Cruz \\ Hospital Ramón y Cajal. Madrid, España. \\ rafamunozcruz@gmail.com
}

María Rodríguez Mármol

Hospital comarcal de Alcañiz. Teruel, España.

Nuria Romero Iglesias

Complejo hospitalario de Jaén. Jaén, España.

\section{RESUMEN}

La transfusión de sangre es una práctica frecuente en el ámbito hospitalario en la que el papel de Enfermería es fundamental, siendo necesarios conocimientos específicos para evitar riesgos y complicaciones. El objetivo de este trabajo fue estudiar los conocimientos y el manejo que tienen un grupo de enfermeras sobre productos hemoderivados. Para ello, se llevó a cabo un estudio descriptivo transversal mediante un cuestionario previamente validado, con 25 ítems y dos opciones de respuesta para cada pregunta. El cuestionario recogía cuestiones sobre conocimientos pre y post transfusionales, así como durante la técnica y en el caso de complicaciones. La media de edad de la muestra fue de 45,23 años, siendo el $45 \%$ hombres. El $44,4 \%$ trabajaba en turno rotatorio, el $68,3 \%$ tenía contrato eventual y el $22,8 \%$ tenía formación específica en hemoderivados. Se realizó un análisis descriptivo de los datos, así como un análisis bivariante en el que se encontraron diferencias significativas en función del sexo, del tipo de contrato, de la experiencia profesional y de la formación específica. En general, los conocimientos sobre hemoderivados son aceptables, aunque existen algunas deficiencias que deberían de ser subsanadas.

Palabras Clave: Actitud, Conocimiento, Enfermería, Sangre, Transfusión Sanguínea.

\section{ABSTRACT}

Blood transfusion is a common practice in the hospital setting in which the role of nursing is essential, specific knowledge being necessary to avoid risks and complications. The aim of this work was to study the knowledge and management on blood products with a group of nurses on blood products. To do this, we conducted a cross-sectional study using a previously validated questionnaire with 25 items and two possible answers to each question. The questionnaire included questions on pre and post transfusion knowledge as well as several actions to take during the technique in the case of complica-
Recibido: 05/04/2016

Aceptado: 15/05/2016 tions. The average age of the sample was 45.23 years, with $45 \%$ o them men. $44.4 \%$ worked in rotating shifts, $68.3 \%$ had temporary contracts and $22.8 \%$ had specific training in blood products. A descriptive analysis of the data was performed, and also a bivariate analysis that found significant differences based on gender, type of contract, professional experience and specific training. In general, knowledge of blood products is acceptable, although there are some shortcomings that should be remedied.

Keywords: Attitudes, Knowledge, Nursing, Blood, Blood Transfusion.

\section{RESUMO}

A transfusão de sangue é uma prática comum no ambiente hospitalar em que o papel da enfermagem é essencial o conhecimento, específica seja necessária para evitar riscos e complicações. O objetivo deste estudo foi o de estudar o conhecimento e gestão com um grupo de enfermeiros sobre produtos derivados do sangue. Para isso, foi realizado um estudo transversal, utilizando questionário previamente validado com 25 itens e duas respostas possíveis a cada pergunta. O questionário incluiu perguntas sobre pré e pós conhecimento transfusão, bem como durante a técnica e, no caso de complicações. A idade média da amostra foi de 45.23 anos, com $45 \%$ dos homens. $44,4 \%$ trabalhavam em Rota, 68,3\% tinham contratos temporários e $22,8 \%$ tinham formação específica em produtos sanguíneos. A análise descritiva dos dados foi realizada, e uma análise bivariada que encontraram diferenças significativas com base no sexo, tipo de contrato, experiência profissional e formação específica. Em geral, o conhecimento de produtos sanguíneos são aceitáveis, embora existam algumas deficiências que devem ser colmatadas.

Palavras- chave: Atitudes, Conhecimentos, Enfermagem, Sangue, Transfusão de Sangue. 


\section{INTRODUCCIÓN}

La sangre siempre ha sido considerada un factor relacionado con la salud y con la fuerza en el ser humano, de ahí que desde el siglo XVII se empiece a intentar realizar transfusiones sanguíneas (1). La transfusión de sangre es el proceso por el que se trasplanta un tejido, siendo en este caso un trasplante de sangre; es un proceso que está sujeto a una legislación rigurosa para establecer un indicador que garantice la seguridad y la calidad de la transfusión (2-4). Tanto la promoción de la donación como la transfusión de sangre es una parte fundamental en el actual sistema de atención sanitaria (5).

La terapia transfusional requiere de sólidos conocimientos para poder planear y ejecutar un plan de trabajo integral para lograr el bienestar de la persona que lo requiera, conocimientos que, según diversos estudios, son insuficientes por parte de los profesionales enfermeros (2, 6-8). El personal de Enfermería que desarrolla su profesión en aspectos relacionados con las transfusiones sanguíneas debe estar en una actualización continua de conocimientos, orientados a desarrollar nuevas técnicas y cuidados enfermeros en este campo, así como medidas de seguridad, ya que es esta categoría profesional la que más accidentes de tipo biológico sufre $(9,10)$. Por otro lado, la aplicación de un protocolo adecuado va a prevenir las serias complicaciones que puedan presentarse en una reacción; dichos protocolos deben estar basados en los principales principios bioéticos $(11,12)$.

La Enfermería cumple un rol fundamental para la recuperación del paciente, ya que es el miembro del equipo de salud que más posibilidad tiene de identificar posibles problemas relacionados con la transfusión (13). El grado de conocimiento del personal enfermero va a influir directamente en la seguridad de la transfusión, por lo que es conveniente la realización de capacitaciones que desarrollen conocimientos, habilidades y competencias, así como la aplicación de instrumentos de evaluación del conocimiento de forma periódica que eviten las causas más frecuentes de muerte relacionadas con la transfusión (14). Entre dichas causas se encuentran la lesión pulmonar aguda, las reacciones hemolíticas y la contaminación bacteriana (15).

\section{OBJETIVOS}

El objetivo de este trabajo es identificar los conocimientos que un grupo de enfermeras y enfermeros que realizan su función asistencial en hospitalización tienen sobre el manejo de hemoderivados. De esta manera se podrán identificar posibles deficiencias y centrar la formación de estos profesionales en corregir dichas deficiencias en una práctica segura.

\section{METOdOLOGÍA}

Se llevó a cabo un estudio descriptivo transversal en el que se encuestaron a 180 profesionales de Enfermería que desarrollaban su profesión en la ciudad de Madrid. No hubo criterios de exclusión, mientras que el único criterio de inclusión fue desarrollar su labor en servicios de hospitalización en dicha ciudad. El muestreo utilizado fue no probabilístico de conveniencia.

Los datos fueron recogidos durante los meses de noviembre de 2015 a enero de 2016 (ambos inclusive) a través de un instrumento autoadministrado para identificar el desempeño en el manejo y administración de hemoderivados, validado con un índice de kappa de 0,8 y extraído de un estudio previo (16). Consta de 25 ítems con dos opciones de respuesta. Además se recogieron variables sociodemográficas como la edad, el sexo, tipo de contrato, turnicidad, experiencia profesional, formación específica y si eran especialistas o generales. Todos los participantes fueron encuestados de forma anónima y voluntaria previo consentimiento informado.

Los datos obtenidos se procesaron con el paquete estadístico SPSS 15 , realizándose cálculos descriptivos a través de media y desviación estándar (DE) para las variables cuantitativas, mientras que para las variables cualitativas se usaron porcentajes y frecuencias. Para el análisis bivariante se utilizó el estadístico Chi-cuadrado. Se consideraron diferencias estadísticamente significativas cuando el valor de significación fue menor a 0,05. En aquellos casos donde las diferencias fueron significativas se llevó a cabo el cálculo del tamaño del efecto a través de la Odds Ratio y su intervalo de confianza al $95 \%$.

\section{RESULTADOS}

La muestra estudiada fue de 180 enfermeros de los cuales el $45 \%$ (81) eran hombres y el $55 \%$ (99) fueron mujeres. La media de edad del total de la muestra fue de $45,23 \pm 10,664$. El $44,4 \%$ (80) trabajaban en turno rotatorio y el resto lo hacía en un turno fijo. El 31,7\% (57) era personal fijo y el 68,3\% (123) eran eventuales. El 78,3\% tenían más de 5 años de experiencia profesional. El 22,8\% (41) tenían formación específica en cuanto al manejo de hemoderivados y el 75,6\% (136) eran enfermeros generales (Tabla 1).

Al analizar descriptivamente el cuestionario se obtuvieron los resultados expuestos en la siguiente tabla (Tabla 2 ).

Se llevó a cabo el análisis bivariante en función de las variables sociodemográficas que se consideraron en el cuestionario, y se obtuvieron las diferencias estadísticamente significativas (Tabla 3).

Tabla 3. Análisis bivariante en función del sexo, tipo de contrato, experiencia profesional y formación específica, a través de la $\mathrm{Chi}^{2}$.

En función del turno que tenían los participantes no se obtuvo ninguna diferencia estadísticamente significativa, al igual que tampoco se encontraron en función de si eran enfermeros generales o especialistas. 


\begin{tabular}{|c|c|c|c|c|}
\hline & Hombres & Mujeres & Total \\
\hline \multicolumn{2}{|c|}{ Sexo } & $45 \%(81)$ & $55 \%(99)$ & $100 \%(180)$ \\
\hline \multirow{2}{*}{ Media de Edad } & $46,30 \pm 10,7$ & $44,36 \pm 10,61$ & $45,23 \pm 10,67$ \\
\hline \multirow{2}{*}{ Turno } & Rotatorio & $38,3 \%(31)$ & $49,5 \%(49)$ & $44,4 \%(80)$ \\
\cline { 2 - 5 } & Fijo & $61,7 \%(50)$ & $50,5 \%(50)$ & $55,6 \%(100)$ \\
\hline \multirow{2}{*}{ Contrato } & Fijo & $37 \%(30)$ & $27,3 \%(27)$ & $31,7 \%(57)$ \\
\cline { 2 - 5 } & Eventual & $63 \%(51)$ & $72,7 \%(72)$ & $68,3 \%(123)$ \\
\hline \multirow{2}{*}{ Experiencia profesional } & Más de 5 años & $77,8 \%(63)$ & $78,8 \%(78)$ & $78,3 \%(141)$ \\
\cline { 2 - 5 } & Menos de 5 años & $22,2 \%(18)$ & $21,2 \%(21)$ & $21,7 \%(39)$ \\
\hline \multirow{2}{*}{ Formación especifica } & Sí & $24,7 \%(20)$ & $21,2 \%(21)$ & $22,8 \%(41)$ \\
\cline { 2 - 5 } & No & $75,3 \%(61)$ & $78,8 \%(78)$ & $77,2 \%(139)$ \\
\hline \multirow{2}{*}{ Especialidad } & Enf. General & $79 \%(64)$ & $72,7 \%(72)$ & $75,6 \%(136)$ \\
\cline { 2 - 5 } & Especialista & $21 \%(17)$ & $27,3 \%(27)$ & $24,4 \%(44)$ \\
\hline
\end{tabular}

Tabla 1. Análisis demográfico segmentado por sexo.

\begin{tabular}{|c|c|c|}
\hline PRE-ADMINISTRACIÓN DE LA TRANSFUSION & $\mathrm{SI}$ & NO \\
\hline 1. La enfermera revisa datos del paciente en la solicitud del producto sanguíneo. & $90 \%(162)$ & $10 \%(18)$ \\
\hline 2. La enfermera recibe y traslada el producto. & $85 \%(153)$ & $15 \%(27)$ \\
\hline 3. La enfermera verifica datos contenidos en la solicitud y etiqueta de la bolsa. & $83,9 \%(151)$ & $16,1 \%(29)$ \\
\hline 4. La enfermera revisa los datos del producto en el módulo de medicamentos. & $83,9 \%(151)$ & $16,1 \%(29)$ \\
\hline 5. Se lava las manos. & $71,7 \%(129)$ & $28,3 \%(51)$ \\
\hline 6. Informa sobre el procedimiento a realizar, observa su estado emocional. & $63,3 \%(114)$ & $36,7 \%(66)$ \\
\hline 7. Toma y registra signos vitales. & $82,2 \%(148)$ & $17,8 \%(32)$ \\
\hline 8. Observa y revisa el estado de la piel. & $64,4 \%(116)$ & $35,6 \%(64)$ \\
\hline 9. Revisa y valora el sitio de punción y calidad de la vía de infusión. & $80,6 \%(145)$ & $19,4 \%(35)$ \\
\hline 10. Realiza anotaciones iniciales en los documentos correspondientes. & $73,9 \%(133)$ & $26,1 \%(47)$ \\
\hline 11. Coteja nuevamente los datos del paciente y del hemoderivado. & $66,1 \%(119)$ & $33,9 \%(61)$ \\
\hline TRAS ADMINISTRACION DE LA TRANSFUSION & $\mathrm{SI}$ & NO \\
\hline 12. Inicia la transfusión y permanece con el paciente los primeros 15 minutos. & $60 \%(108)$ & $40 \%(72)$ \\
\hline 13. Registra sus signos vitales. & $71,1 \%(128)$ & $28,9 \%(52)$ \\
\hline 14. Revisa la respuesta del paciente a la transfusión cada 30 minutos. & $26,1 \%(47)$ & $73,9 \%(133)$ \\
\hline NO SE SUSPENDE POR COMPLICACIONES & $\mathrm{SI}$ & NO \\
\hline 15. Hace los registros en los formatos correspondientes. & $69,4 \%(125)$ & $30,6 \%(55)$ \\
\hline 16. Valora clínicamente el estado del paciente. & $56,7 \%(102)$ & $43,3 \%(78)$ \\
\hline 17. Envía tarjeta de control del producto sanguíneo al banco de sangre. & $82,2 \%(148)$ & $17,8 \%(32)$ \\
\hline 18. Evaluación del grado de satisfacción del paciente. & $68,3 \%(123)$ & $31,7 \%(57)$ \\
\hline 19. Logro del objetivo terapéutico. & $87,8 \%(158)$ & $12,2 \%(22)$ \\
\hline SE SUSPENDE POR COMPLICACIONES & SI & NO \\
\hline 20. Reacciones adversas por la transfusión, suspender la transfusión. & $94,4 \%(170)$ & $5,6 \%(10)$ \\
\hline 21. Avisar al médico. & $92,2 \%(166)$ & $7,8 \%(14)$ \\
\hline 22. Registrar los signos vitales. & $94,4 \%(170)$ & $5,6 \%(10)$ \\
\hline 23. Observación y control de reacciones adversas. & $82,8 \%(149)$ & $17,2 \%(31)$ \\
\hline 24. Toma muestra sanguínea. & $97,8 \%(176)$ & $2,2 \%(4)$ \\
\hline 25. Hace registro en los formatos correspondientes. & - & $100 \%(180)$ \\
\hline
\end{tabular}

Tabla 2. Frecuencias obtenidas en cada ítem. 


\begin{tabular}{|c|c|c|c|}
\hline \multicolumn{4}{|c|}{ En función del sexo } \\
\hline & Valor de $\mathrm{p}$ & OR & IC 95\% \\
\hline Ítem 11 & 0,038 & 0,518 & {$[0,27-0,96]$} \\
\hline Ítem 14 & 0,036 & 0,476 & {$[0,22-0,95]$} \\
\hline Ítem 18 & 0,041 & 0,517 & {$[0,27-0,97]$} \\
\hline \multicolumn{4}{|c|}{ En función del tipo de contrato } \\
\hline & Valor de $\mathrm{p}$ & OR & IC $95 \%$ \\
\hline Ítem 11 & 0,003 & 0,380 & {$[0,19-0,73]$} \\
\hline Ítem 16 & 0,001 & 3,332 & {$[1,65-6,70]$} \\
\hline Ítem 18 & 0,000 & 0,285 & {$[0,14-0,55]$} \\
\hline \multicolumn{4}{|c|}{ En función de la experiencia profesional } \\
\hline & Valor de $p$ & OR & IC 95\% \\
\hline Ítem 3 & 0,035 & 0,228 & {$[0,05-0,99]$} \\
\hline Ítem 18 & 0,037 & 0,398 & {$[0,16-0,96]$} \\
\hline \multicolumn{4}{|c|}{ En función formación específica } \\
\hline & Valor de $\mathrm{p}$ & OR & IC 95\% \\
\hline Ítem 2 & 0,016 & 0,355 & {$[0,14-0,84]$} \\
\hline Ítem 23 & 0,017 & 5,141 & {$[1,17-22,55]$} \\
\hline
\end{tabular}

Nota: el contenido de los ítems se puede consultar en la Tabla 2.

\section{DISCUSIÓN}

Los resultados arrojados en el análisis descriptivo muestran datos llamativos como que casi el $30 \%$ de los encuestados no realiza lavado de manos previo a la transfusión de hemoderivados, que más del $35 \%$ no informa al paciente del proceso que se va a llevar a cabo, que el $40 \%$ no está observando al paciente durante los primeros 15 minutos de transfusión y que más del $70 \%$ no lo hace después. Estas acciones pueden ser derivadas de la falta de tiempo por parte del personal de Enfermería o por el desconocimiento sobre este tipo de prácticas. Es conveniente comparar los resultados obtenidos con los de Cando, Calle y Morales en 2014, donde el $81 \%$ de los sanitarios encuestados afirma realizar el lavado de manos antes de manejar hemoderivados; sin embargo, en el mismo estudio casi un $56 \%$ no observan la reacción del paciente durante los primeros 15 minutos (10). Respecto a la vigilancia después de la transfusión, en el estudio de Tigua del año 2014 un tercio de las enfermeras no la realizan, es decir, menos de la mitad que en el actual estudio (7). En el lado contrario, hay que decir que los conocimientos en el caso de que la transfusión se suspenda por complicaciones, los profesionales tienen un manejo adecuado, ya que la inmensa mayoría de los profesionales declaran hacer las actuaciones adecuadas, mostrando una actitud similar a la obtenida en la muestra de Mendoza, Moreno y Sequera (2).

En el análisis bivariante se hallaron diferencias significativas en función del sexo de los participantes, siendo las mujeres las que cotejan nuevamente los datos del paciente y del producto antes de la transfusión, revisan la respuesta del paciente y evalúan el grado de satisfacción después de la realización de la técnica. En función del tipo de contrato de las enfermeras, aquellos que tenían un contrato eventual fueron los que co- tejaban los datos de paciente y producto y evaluaban el grado de satisfacción en mayor proporción que aquellos con contrato fijo, siendo éstos los que valoraban, en mayor proporción, el estado del paciente tras la transfusión. No ocurre lo mismo en el estudio llevado a cabo por Flores-Torrecillas y cols. en 2014, donde el tipo de contrato no influye significativamente en los conocimientos de los profesionales (16).

Dependiendo de la experiencia laboral, aquellos que tenían menos de 5 años de experiencia fueron los que más verificaban el contenido de la petición, de la etiqueta de la bolsa y evaluaban el grado de satisfacción del paciente. El hecho de que los profesionales con más experiencia tengan un mejor manejo de los hemoderivados se afirma también en el estudio llevado a cabo en 2013 por Gómez, Quevedo y Calderón, donde se hace especial mención a los consejos de los profesionales a las personas antes de donar sangre (5). Por último, se encontraron diferencias en función de la formación específica, siendo aquellos que la tienen los que revisaban las reacciones adversas en mayor proporción que los que no, mientras que éstos se encargaban en mayor proporción de recibir y trasladar el producto. Resultados equiparables a los obtenidos por Lumenia, Barichello, de Mattia y Barbosa, donde el hecho de haber participado en un curso específico de formación mostró diferencias significativas hacia la mejora en los conocimientos sobre transfusiones sanguíneas (14).

A diferencia de los resultados obtenidos por Camacho en 2010, donde los profesionales que desarrollaban su labor en el turno de noche fueron los que presentaron menor nivel de conocimientos, en el presente estudio no se encontraron diferencias significativas en función del turno que desempeñaban los enfermeros, al igual que tampoco en función del hecho de tener 
un título de especialista; respecto a este título, el estudio llevado a cabo por Velasco-Rodríguez, Osorio-Cruz, Peguero-Ortiz y Mora-Brambila en 2013 sí que encontró diferencias en función de la posesión de la especialidad $(9,15)$.

\section{CONSIDERACIONES FINALES}

Al tratarse de un estudio descriptivo transversal con un muestreo no probabilístico, no se puede medir temporalidad ni se pueden extrapolar los resultados a otras poblaciones. Se puede concluir afirmando que, en general, la muestra estudiada presenta un buen manejo en la transfusión de hemoderivados, aunque existen algunas deficiencias en determinadas prácticas, ya sea por desconocimiento o por falta de tiempo. Sería conveniente formar al profesional de Enfermería específicamente en el manejo de hemoderivados, ya que es una práctica habitual en el ámbito clínico, aunque también sería adecuado un incremento del personal enfermero; estas dos acciones disminuirían los posibles errores o complicaciones relacionados con esta práctica. Como propuesta de estudio para el futuro resultaría interesante utilizar este estudio como base para la realización de investigaciones que dispongan de más recursos y de una financiación que posibilite la generalización a otras poblaciones mayores, de manera que se pueda incidir en los aspectos en los que los profesionales tengan más deficiencias sobre el manejo y los conocimientos de hemoderivados.

\section{REFERENCIAS BIBLIOGRÁFICAS}

1. Tovar D, Mejías M, Rodríguez L. Cuidados de enfermería a pacientes con falla multiorgánica que reciben hemoderivados. Enfermería Global. 2011;10(23): 41-52.

2. Mendoza L, Moreno J, Sequera J. Información que poseen los profesionales de Enfermería de atención directa sobre solicitud, transporte y administración de hemoderivados. Hospital materno infantil "Dr. José María Vargas" [tesis]. Valencia (estado Carabobo): Universidad Central de Venezuela; 2006.

3. Quevedo SS. Evaluación de los conocimientos en el manejo de los hemoderivados en las dicentes de tercer año de la Escuela de Enfermería de la Universidad de Guayaquil y propuesta de un manual de técnicas y procedimientos en la administración de hemoderivados [tesis]. Guayaquil: Universidad de Guayaquil; 2014. Disponible en: http://repositorio.ug.edu.ec/bitstream/ redug/3780/1/TESIS\%20DE\%20GRADO\%20EVALUACION\%20DE\%20LOS\%20CONOCIMIENTOS\%20EN\%20 LA\%20ADMINISTRACION\%20DE\%20LOS\%2OHEMODERIVADOS\%20EN\%20LAS\%20D.pdf

4. Velázquez D, Guerrero D. El recorrido de la sangre: enfermería de principio a fin. Hygia. 2009;16 (70):4 4-47.

5. Gómez DA, Quevedo LM, Calderón P. Conocimientos y prácticas en el proceso de donación de sangre, del personal de salud que labora en los bancos de sangre del departamento del Huila, para el periodo 2013-b [Internet]. 2013 [citado 21 Abr 2016]. Disponible en: https://contenidos.usco.edu.co/images/documentos/
grados/T.G.Enfermeria/226.T.G-Diego-Andres-Gomez,Lina-Maria-Quevedo,-Paula-Calderon-Celis-2013.pdf

6. Valderrama ML, Malpica FN, Franco KY. Cuidado de Enfermería en la administración de hemoderivados. Revista Cuidarte. 2015;6 (1): 955-963.

7. Tigua MC. Intervención de enfermería en la administración de hemocomponente en adulto obstétrico en el área de $\mathrm{UCl}$ del Hospital Gineco-Obstétrico Enrique C. Sotomayor de diciembre del 2013 al 30 mayo de 2014 [tesis]. Guayaquil: Universidad Católica de Santiago de Guayaquil; 2014

8. López B. Análisis del conocimiento enfermero ante una transfusión sanguínea: revisión bibliográfica. Recién. $2015 ; 10$.

9. Velasco-Rodríguez R, Osorio-Cruz $A D$, Peguero-Ortiz RE, Mora-Brambila AB. Cumplimiento en el manejo de hemoderivados por personal de enfermería en un hospital de México. Rev Enferm Inst Mex Seguro Soc. 2013; 21(1):15-21.

10. Cando EK, Calle JA, Morales RF. Conocimientos, actitudes y prácticas sobre las normas de bioseguridad en el manejo y administración de sangre y hemoderivados por el personal de enfermería en el departamento de pediatría del Hospital" Vicente Corral Moscoso" [tesis]. Cuenca (Ecuador): Universidad de Cuenca; 2014. Disponible en: http://dspace.ucuenca.edu.ec/bitstream/123456789/20905/1/Tesis.pdf

11. Merchán N, Rojo RM, Carrero A, Rodríguez-Arias CM, Blas MJ. Actuación de Enfermería ante una transfusión de sangre y derivados. Cáceres: Universidad de Extremadura; 1996. Disponible en: https://ruidera. uclm.es/xmlui/bitstream/handle/10578/312/1996-6. pdf?sequence $=1$ \&isAllowed $=y$

12. Abreu Y, Rodríguez H. Principios bioéticos aplicados por el profesional de enfermería en la administración de transfusiones sanguíneas y sus componentes en la unidad clínica de emergencia de adultos del Hospital "Dr. Domingo Luciani" en el primer semestre del año 2010 [tesis]. Caracas: Universidad central de Venezuela; 2015.

13. Valencia BM, Naranjo LE. Adherencias a las guías cateterismo vesical y aplicación de hemoderivados por el personal de Enfermería. Medellín: Universidad del CES; 2012. Disponible en: http://bdigital.ces.edu.co:8080/ repositorio/bitstream/10946/291/2/Microsoft $\% 20$ Word\%20-\%20TESIS.pdf

14. Lumênia J, Barichello E, de Mattia AL, Barbosa MH. Factores asociados al conocimiento del equipo de enfermería sobre transfusión sanguínea en un hospital de enseñanza. Rev Latino-Am Enfermagen. 2015; 23(4): 595-602.

15. Camacho T. Competencias profesionales de enfermería en la atención del paciente sometido a terapia transfusional [tesis]. San Nicolás de los Garza: Universidad Autónoma de Nuevo León; 2010. Disponible en: http:// eprints.uanl.mx/5679/1/1080192147.PDF

16. Flores-Torrecillas R, Carballo-Monreal MR, Alvarez-Villaseñor AS, Valdez-Márquez ML, González-Ojeda A, FuentesOrozco C. Manejo y administración de hemoderivados por personal de enfermería en un hospital de segundo nivel. Enfermería universitaria. 2014;11(3): 94-100.- 